\title{
Linearization method in classification problems of linear algebra
}

\author{
Vladimir V. Sergeichuk \\ Institute of Mathematics, Tereshchenkivska 3, Kiev, Ukraine \\ E-mail address: sergeich@imath.kiev.ua
}

\begin{abstract}
The author devised in [Math. USSR-Izv. 31 (1988) 481501] a procedure that reduces the problem of classifying systems of forms and linear mappings to the problem of classifying systems of linear mappings. We give a brief exposition of this method, review results that were obtained by using this method (and were published mainly in Russian), and give examples of classification problems that can be solved by this method.
\end{abstract}

\section{Introduction}

The theory of bilinear forms over $\mathbb{C}$ reduces to the theory of matrix pencils due to the following trivial corollary from Theorem 3 in $[30, \S 95]$ :

square complex matrices $M$ and $N$ are congruent if and only if the pairs $\left(M, M^{T}\right)$ and $\left(N, N^{T}\right)$ are simultaneously

equivalent,

that is, $M=S^{T} N S$ for some nonsingular $S$ if and only if $M=P N Q$ and $M^{T}=P N^{T} Q$ for some nonsingular $P$ and $Q$. Roiter [41] extended (1) to systems of linear mappings and bilinear forms over an algebraically closed field of characteristic different from 2 .

Basing on Roiter's ideas, the author devised in $[45,46,47,49]$ a procedure (we call it the linearization method) that reduces the problem of classifying an arbitrary system of linear mappings and bilinear/sesquilinear

1991 Mathematics Subject Classification. 15A21.

Key words: Bilinear and sesquilinear forms, Congruence, Canonical matrices.

The research was done while the author was visiting the University of São Paulo supported by FAPESP, processo 05/59407-6. 
forms over a field or skew field $\mathbb{F}$ of characteristic not 2 with involution (possibly, the identity) to the the problems of classifying

- some system of linear mappings and

- Hermitian forms over finite extensions of the center of $\mathbb{F}$.

The linearization method is presented in Section 2. In particular, it implies (see Corollary 2.3) that each system of forms and linear mappings over $\mathbb{C}, \mathbb{R}$, or quaternions decomposes into a direct sum of indecomposable systems uniquely up to isomorphism of summands. Hence, it suffices to classify only indecomposable systems. Many authors proved this statement for special classes of systems of forms and linear mappings in the process of their classification.

In Section 3 we formulate some results obtained by the linearization method in $[45,46,47,49]([45,46,47]$ are practically unknown since were published in Russian). We also give examples of classification problems that were solved or can be solved by this method.

In Section 4 we consider systems of tensors and extend the linearization method to $n$-linear forms with $n \geqslant 2$.

In Section 5 we present an analogous method that reduces the problem of classifying systems of linear and semilinear mappings to the problem of classifying systems of linear mappings.

\section{Linearization method for systems of forms and linear map- pings}

Classification problems for systems of linear mappings can be formulated in terms of quivers (i.e., oriented graphs) and their representations introduced by Gabriel [11, 13]. This notion was extended in [45] to representations of graphs with nonoriented, oriented, and doubly oriented $(\longleftrightarrow)$ edges, which admits to formulate classification problems for systems of forms and linear mappings. This section contains a brief summary of some results about representations of such graphs.

We denote by $\mathbb{F}$ a field or skew field with involution $a \mapsto \bar{a}$, that is, a bijection $\mathbb{F} \rightarrow \mathbb{F}$ satisfying $\overline{a+b}=\bar{a}+\bar{b}, \overline{a b}=\bar{b} \bar{a}$, and $\overline{\bar{a}}=a$ for all $a, b \in \mathbb{F}$. All vector spaces over $\mathbb{F}$ are assumed to be finite dimensional right vector spaces. A mapping $B: U \times V \rightarrow \mathbb{F}$ on vector spaces $U$ and $V$ over $\mathbb{F}$ is called a sesquilinear form if

$$
\begin{aligned}
B\left(u a+u^{\prime} a^{\prime}, v\right) & =\bar{a} B(u, v)+\bar{a}^{\prime} B\left(u^{\prime}, v\right), \\
B\left(u, v a+v^{\prime} a^{\prime}\right) & =B(u, v) a+B\left(u, v^{\prime}\right) a^{\prime}
\end{aligned}
$$

São Paulo J.Math.Sci. 1, 2 (2007), 219-240 
for all $u, u^{\prime} \in U, v, v^{\prime} \in V$, and $a, a^{\prime} \in \mathbb{F}$. This form is bilinear if $\mathbb{F}$ is a field and the involution $a \mapsto \bar{a}$ is the identity.

A dograph (double ordered graph) is a graph with nonoriented, oriented, and double oriented edges; for example,

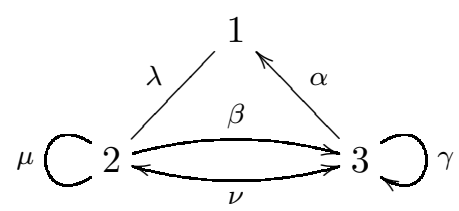

We suppose that the vertices of dographs are $1,2, \ldots, n$, and that the number of edges between two vertices can be arbitrary.

A representation $\mathcal{A}$ of a dograph $P$ over $\mathbb{F}$ is given by assigning to each vertex $i$ a vector space $\mathcal{A}_{i}$ over $\mathbb{F}$, to each arrow $\alpha: i \rightarrow j$ a linear mapping $\mathcal{A}_{\alpha}: \mathcal{A}_{i} \rightarrow \mathcal{A}_{j}$, to each nonoriented edge $\beta: i-j(i \leqslant j)$ a sesquilinear form $\mathcal{A}_{\beta}: \mathcal{A}_{i} \times \mathcal{A}_{j} \rightarrow \mathbb{F}$, and to each doubly oriented edge $\gamma: i \longleftrightarrow j(i \leqslant j)$ a sesquilinear form $\mathcal{A}_{\gamma}: \mathcal{A}_{i}^{*} \times \mathcal{A}_{j}^{*} \rightarrow \mathbb{F}$ on the ${ }^{*}$ dual vector spaces. By the *dual space to a vector space $V$ we mean the space $V^{*}$ of all mappings $\varphi: V \rightarrow \mathbb{F}$ that are semilinear, this means that

$$
\varphi(u a+v b)=\bar{a} \varphi(u)+\bar{b} \varphi(v), \quad u, v \in V, \quad a, b \in \mathbb{F} .
$$

For every linear mapping $A: U \rightarrow V$, we define the ${ }^{*}$ adjoint mapping $A^{*}: V^{*} \rightarrow U^{*}$ by putting $A^{*} \varphi:=\varphi A$ for all $\varphi \in V^{*}$.

The vector

$$
\operatorname{dim} \mathcal{A}:=\left(\operatorname{dim} \mathcal{A}_{1}, \ldots, \operatorname{dim} \mathcal{A}_{n}\right)
$$

is called the dimension of $\mathcal{A}$.

For example, each representation of the dograph (2) is a system

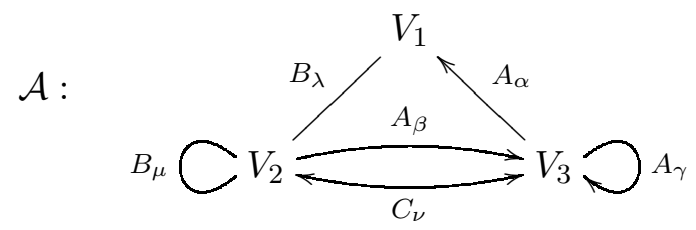

of vector spaces $V_{1}, V_{2}, V_{3}$ over $\mathbb{F}$, linear mappings $A_{\alpha}: V_{3} \rightarrow V_{1}, A_{\beta}: V_{2} \rightarrow$ $V_{3}, A_{\gamma}: V_{3} \rightarrow V_{3}$, and forms $B_{\lambda}: V_{1} \times V_{2} \rightarrow \mathbb{F}, B_{\mu}: V_{2} \times V_{2} \rightarrow \mathbb{F}, C_{\nu}: V_{2}^{*} \times$ $V_{3}^{*} \rightarrow \mathbb{F}$. 
A morphism $f=\left(f_{1}, \ldots, f_{n}\right): \mathcal{A} \rightarrow \mathcal{A}^{\prime}$ of representations of $D$ is a set of linear mappings $f_{i}: \mathcal{A}_{i} \rightarrow \mathcal{A}_{i}^{\prime}$ suth that

$$
f_{j} \mathcal{A}_{\alpha}=\mathcal{A}_{\alpha}^{\prime} f_{i}, \quad \mathcal{A}_{\beta}(x, y)=\mathcal{A}_{\beta}^{\prime}\left(f_{i} x, f_{j} y\right), \quad \mathcal{A}_{\gamma}\left(x f_{i}, y f_{j}\right)=\mathcal{A}_{\gamma}^{\prime}(x, y)
$$

for all edges $\alpha: i \longrightarrow j, \beta: i-j(i \leqslant j)$, and $\gamma: i \longleftrightarrow j(i \leqslant j)$. A morphism $f$ is an isomorphism if all $f_{i}$ are bijections. A morphism or isomorphism $f: \mathcal{A} \rightarrow \mathcal{A}^{\prime}$ is called an endomorphism or isomorphism, respectively, if $\mathcal{A}=\mathcal{A}^{\prime}$. The composition of two morphisms is a morphism.

The direct sum $\mathcal{A} \oplus \mathcal{A}^{\prime}$ of representations $\mathcal{A}$ and $\mathcal{A}^{\prime}$ of $P$ is the representation consisting of the direct sums of the corresponding vector spaces and the direct sums of the corresponding linear mappings and forms.

For every dograph $D$, we denote by $\underline{D}$ the quiver with involution on the set of vertices and on the set of arrows obtained from $D$ by replacing

- each vertex $i$ of $D$ by the vertices $i$ and $i^{*}$,

- each arrow $\alpha: i \rightarrow j$ by the arrows $\alpha: i \rightarrow j$ and $\alpha^{*}: j^{*} \rightarrow i^{*}$,

- each edge $\beta: i-j(i \leqslant j)$ by $\beta: j \rightarrow i^{*}$ and $\beta^{*}: i \rightarrow j^{*}$,

- each edge $\gamma: i \longleftrightarrow j(i \leqslant j)$ by $\gamma: j^{*} \rightarrow i$ and $\gamma^{*}: i^{*} \rightarrow j$.

For example,

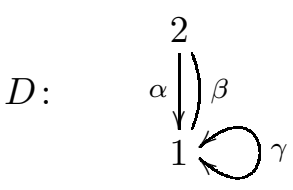

$\underline{D}:$

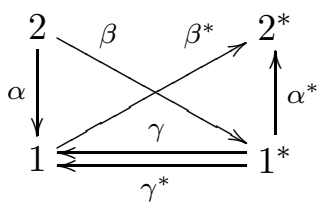

Respectively, for each representation $\mathcal{M}$ of $D$ over $\mathbb{F}$, we define the representation $\underline{\mathcal{M}}$ of $\underline{D}$ by replacing

- each vector space $V$ in $\mathcal{M}$ by the pair of spaces $V$ and $V^{*}$,

- each linear mapping $A: U \rightarrow V$ by the mutually *adjoint mappings $A: U \rightarrow V$ and $A^{*}: V^{*} \rightarrow U^{*}$,

- each sesquilinear form $B: V \times U \rightarrow \mathbb{F}$ by the mutually *adjoint mappings $B: u \in U \mapsto B(?, u) \in V^{*}$ and $B^{*}: v \in V \mapsto \overline{B(v, ?)} \in$ $U^{*}$.

- each sesquilinear form $C: V^{*} \times U^{*} \rightarrow \mathbb{F}$ by the mutually *adjoint mappings $C: u^{*} \in U^{*} \mapsto C\left(?, u^{*}\right) \in V^{* *}=V$ and $C^{*}: v^{*} \in V^{*} \mapsto$ $\overline{C\left(v^{*}, ?\right)} \in U$. 
For example, for representations of (4) we have

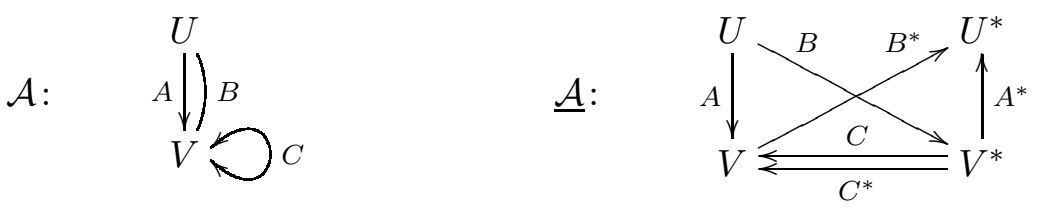

For every (usual) representation $\mathcal{M}$ of $\underline{D}$ we define the dual representation $\mathcal{M}^{\circ}$ of $\underline{D}$ consisting of the vector spaces $\mathcal{M}_{v}^{\circ}:=\mathcal{M}_{v^{*}}^{*}$ and the linear mappings $\mathcal{M}_{\alpha}^{\circ}:=\mathcal{M}_{\alpha^{*}}^{*}$ for all vertices $v$ and arrows $\alpha$ of $\underline{D}$. For example, for representations of the quiver $\underline{D}$ defined in (4),

$\mathcal{M}:$
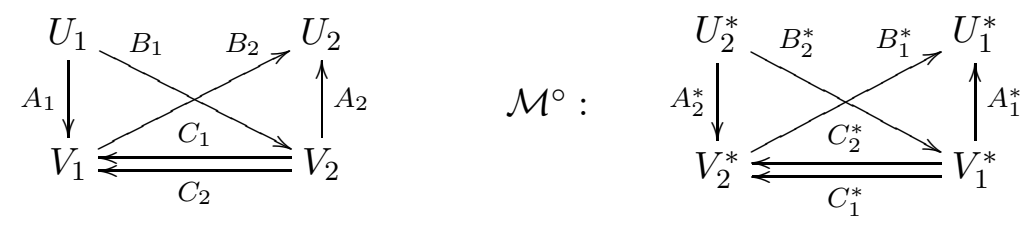

The second representation in (5) is selfdual: $\underline{\mathcal{A}}^{\circ}=\underline{\mathcal{A}}$.

In a similar way, for each morphism $f: \mathcal{M} \rightarrow \mathcal{N}$ of representations of $\underline{D}$ we construct the dual morphism $f^{\circ}: \mathcal{N}^{\circ} \rightarrow \mathcal{M}^{\circ}$ by putting $f_{i}^{\circ}:=f_{i^{*}}^{*}$ for all vertices $i$ of $\underline{D}$. An isomorphism $f: \mathcal{M} \simeq \mathcal{N}$ of selfdual representations $\mathcal{M}$ and $\mathcal{N}$ is called a congruence if $f^{\circ}=f^{-1}$. Two representations $\mathcal{A}$ and $\mathcal{B}$ of a dograph $D$ are isomorphic if and only if the corresponding selfdual representations $\underline{\mathcal{A}}$ and $\underline{\mathcal{B}}$ of the quiver $\underline{D}$ are congruent.

A relation on a quiver $Q$ over a field or skew field $\mathbb{F}$ is a formal expression of the form

$$
\sum_{i=1}^{m} c_{i} \alpha_{i p_{i}} \cdots \alpha_{i 2} \alpha_{i 1}=0
$$

in which $c_{i}$ are nonzero elements of the center of $\mathbb{F}$ and

$$
u \stackrel{\alpha_{i 1}}{\longrightarrow} u_{i 2} \stackrel{\alpha_{i 2}}{\longrightarrow} \cdots \stackrel{\alpha_{i, p_{i}-1}}{\longrightarrow} u_{i p_{i}} \stackrel{\alpha_{i p_{i}}}{\longrightarrow} v
$$

are oriented paths on $Q$ with the same initial vertex $u$ and the same final vertex $v$. A path may have length 0 if $u=v$; it is replaced by 1 in (6). Therefore, if $u=v$ then (6) may have ' 1 ' instead of ' 0 ' in its right-hand side. A representation $\mathcal{A}$ of $Q$ satisfies the relation (6) if

$$
\sum_{i=1}^{m} c_{i} \mathcal{A}_{\alpha_{i p_{i}}} \cdots \mathcal{A}_{\alpha_{i 2}} \mathcal{A}_{\alpha_{i 1}}=0 .
$$


By a dograph with relations, we mean a dograph $D$ with a finite set of relations on its quiver with involution $\underline{D}$, and consider only those representations $\mathcal{A}$ of $D$ for which the corresponding selfdual representations $\underline{\mathcal{A}}$ of $\underline{D}$ satisfy these relations. Clearly, if $\underline{\mathcal{A}}$ satisfies the relation (6), then it satisfies also the adjoint relation

$$
\sum_{i=1}^{m} \bar{c}_{i} \alpha_{i 1}^{*} \alpha_{i 2}^{*} \cdots \alpha_{i p_{i}}^{*}=0
$$

If $D$ is a dograph with relations, then we consider $\underline{D}$ as the quiver with relations whose set of relations consists of the relations of $D$ and the adjoint relations.

Suppose we know any maximal set ind $(\underline{D})$ of nonisomorphic indecomposable representations of the quiver $\underline{D}$ (this means that every indecomposable representation of $\underline{D}$ is isomorphic to exactly one representation from $\operatorname{ind}(\underline{D}))$. We define the following sets $\operatorname{ind}_{0}(\underline{D})$ and $\operatorname{ind}_{1}(\underline{D})$ :

- we replace each representation in $\operatorname{ind}(\underline{D})$ that is isomorphic to a selfdual representation by one that is actually selfdual, i.e., has the form $\underline{\mathcal{A}}$, and denote the set of these $\underline{\mathcal{A}}$ by $\operatorname{ind}_{0}(\underline{D})$, and then

- in each of one- or two-element subsets $\{\mathcal{M}, \mathcal{L}\} \subset \operatorname{ind}(\underline{D}) \backslash \operatorname{ind}_{0}(\underline{D})$ satisfying $\mathcal{M}^{\circ} \simeq \mathcal{L}$, we select one representation and denote the set of selected representations by $\operatorname{ind}_{1}(\underline{D})$.

(If $\mathcal{M} \sim \mathcal{M}^{\circ}$, then $\{\mathcal{M}, \mathcal{L}\}$ consists of one representation, and we take it.) We obtain a new set ind $(\underline{D})$ partitioned into 3 subsets:

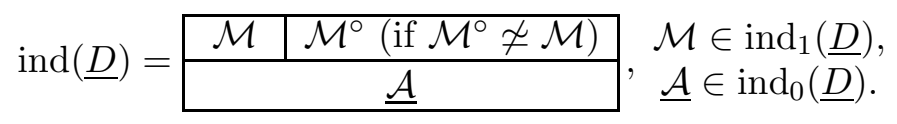

For each representation $\mathcal{M}$ of $\underline{D}$, we define the representation $\mathcal{M}^{+}$of $D$ by putting $\mathcal{M}_{i}^{+}:=\mathcal{M}_{i} \oplus \mathcal{M}_{i^{*}}^{*}$ for all vertices $i$ of $D$ and

$$
\mathcal{M}_{\alpha}^{+}:=\left[\begin{array}{cc}
\mathcal{M}_{\alpha} & 0 \\
0 & \mathcal{M}_{\alpha^{*}}^{*}
\end{array}\right], \quad \mathcal{M}_{\beta}^{+}:=\left[\begin{array}{cc}
0 & \mathcal{M}_{\beta^{*}}^{*} \\
\mathcal{M}_{\beta} & 0
\end{array}\right], \quad \mathcal{M}_{\gamma}^{+}:=\left[\begin{array}{cc}
0 & \mathcal{M}_{\gamma} \\
\mathcal{M}_{\gamma^{*}}^{*} & 0
\end{array}\right]
$$

for all edges $\alpha: i \longrightarrow j, \beta: i-j(i \leqslant j)$, and $\gamma: i \longleftrightarrow j(i \leqslant j)$.

For every representation $\mathcal{A}$ of $D$ and for every selfdual automorphism $f=f^{\circ}: \underline{\mathcal{A}} \stackrel{\sim}{\rightarrow} \underline{\mathcal{A}}$, we denote by $\mathcal{A}^{f}$ the representation of $D$ obtained from 
$\mathcal{A}$ by replacing each form $\mathcal{A}_{\beta}(\beta: i-j, i \leqslant j)$ by $\mathcal{A}_{\beta}^{f}:=\mathcal{A}_{\beta} f_{j}$ and each form $\mathcal{A}_{\gamma}(\gamma: i \longleftrightarrow j, i \leqslant j)$ by $\mathcal{A}_{\gamma}^{f}:=f_{i}^{-1} \mathcal{A}_{\gamma}$.

Let $\operatorname{ind}(\underline{D})$ be partitioned as in (8), and let $\underline{\mathcal{A}} \in \operatorname{ind}_{0}(\underline{D})$. By $[49$, Lemma 1], the set $R$ of noninvertible elements of the endomorphism ring $\operatorname{End}(\underline{\mathcal{A}})$ is the radical. Therefore, $\mathbb{T}(\mathcal{A}):=\operatorname{End}(\underline{\mathcal{A}}) / R$ is a field or skew field, on which we define the involution

$$
(f+R)^{\circ}:=f^{\circ}+R .
$$

For each nonzero $a=a^{\circ} \in \mathbb{T}(\mathcal{A})$, we fix a selfdual automorphism $f_{a}=$ $f_{a}^{\circ} \in a$, and define $\mathcal{A}^{a}:=\mathcal{A}^{f_{a}}$ (one can take $f_{a}:=\left(f+f^{\circ}\right) / 2$ for any $f \in a$ ). The set of representations $\mathcal{A}^{a}$ is called the orbit of $\mathcal{A}$.

For each Hermitian form

$$
\varphi(x)=x_{1}^{\circ} a_{1} x_{1}+\cdots+x_{r}^{\circ} a_{r} x_{r}, \quad 0 \neq a_{i}=a_{i}^{\circ} \in \mathbb{T}(\mathcal{A}),
$$

we write

$$
\mathcal{A}^{\varphi(x)}:=\mathcal{A}^{a_{1}} \oplus \cdots \oplus \mathcal{A}^{a_{r}}
$$

The following theorem was proved in [49, Theorem 1].

Theorem 2.1. Over a field or skew field $\mathbb{F}$ of characteristic different from 2 with involution $a \mapsto \bar{a}$, every representation of a dograph $D$ with relations is isomorphic to a direct sum

$$
\mathcal{M}_{1}^{+} \oplus \cdots \oplus \mathcal{M}_{p}^{+} \oplus \mathcal{A}_{1}^{\varphi_{1}(x)} \oplus \cdots \oplus \mathcal{A}_{q}^{\varphi_{q}(x)}
$$

where

$$
\mathcal{M}_{i} \in \operatorname{ind}_{1}(\underline{D}), \quad \underline{\mathcal{A}}_{j} \in \operatorname{ind}_{0}(\underline{D})
$$

and $\mathcal{A}_{j} \neq \mathcal{A}_{j^{\prime}}$ if $j \neq j^{\prime}$. This sum is determined by the original representation uniquely up to permutation of summands and replacement of $\mathcal{A}_{j}^{\varphi_{j}(x)}$ by $\mathcal{A}_{j}^{\psi_{j}(x)}$, where $\varphi_{j}(x)$ and $\psi_{j}(x)$ are equivalent Hermitian forms over $\mathbb{T}\left(\mathcal{A}_{j}\right)$ with involution (9).

Recall that a real closed field is a field whose algebraic closure has degree 2. Theorem 2.1 ensures the following generalization of the law of inertia for quadratic forms (the corresponding Theorem 2 in [49] is formulated incorrectly in the case of representations over quaternions).

Theorem 2.2. Let $\mathbb{F}$ be either 
(i) an algebraically closed field of characteristic different from 2 with the identity involution, or

(ii) an algebraically closed field with nonidentity involution, or

(iii) a real closed field or the skew field of quaternions over a real closed field.

Then every representation of a dograph $D$ with relations over $\mathbb{F}$ is isomorphic to a direct sum, uniquely determined up to permutation of summands, of representations of the following form (with $\mathcal{M} \in \operatorname{ind}_{1}(\underline{D})$ and $\left.\underline{\mathcal{A}} \in \operatorname{ind}_{0}(\underline{D})\right)$, respectively:

(i) $\mathcal{M}^{+}, \mathcal{A}$

(ii) $\mathcal{M}^{+}, \mathcal{A}, \mathcal{A}^{-}$,

(iii) $\mathcal{M}^{+},\left\{\begin{aligned} & \mathcal{A}, \text { if } \mathbb{T}(\mathcal{A}) \text { is an algebraically closed field with the } \\ & \text { identity involution or a skew field of quaternions } \\ & \text { with involution different from the quaternionic } \\ & \text { conjugation, and }\end{aligned}\right.$

Corollary 2.3. Each system of linear mappings and bilinear/sesquilinear forms on vector spaces over $\mathbb{R}, \mathbb{C}$, and $\mathbb{H}$ decomposes into a direct sum of indecomposable systems uniquely up to isomorphisms of summands.

\section{Applications of the linearization method}

\subsection{Classification problems that were solved by the linearization method}

The problems of classifying representations of the dographs

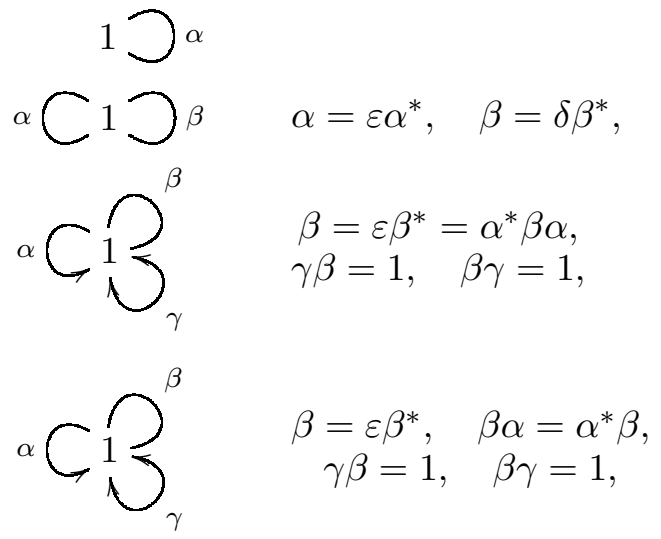


in which $\varepsilon, \delta \in\{-1,1\}$ (the edges $\gamma$ and the relations $\gamma \beta=1, \beta \gamma=1$ ensure the nonsingularity of the form assigned to $\beta$ in each representation) are the problems of classifying, respectively:

- sesquilinear forms, studied in [12, 38, 39, 43, 44, 48],

- pairs of forms, in which the first is $\varepsilon$-Hermitian and the second is $\delta$-Hermitian, studied in $[25,27,28,35,42,44,52,53,54,55]$ (see also the bibliography of 225 items in [52]),

- isometric operators on a space with nonsingular $\varepsilon$-Hermitian form, studied in [1, 2, 25, 26, 29, 40,44],

- selfadjoint operators on a space with nonsingular $\varepsilon$-Hermitian form, studied in $[1,2,21,22,26,34,35,36,40,44,55]$.

An operator $A$ is said to be isometric or selfadjoint with respect to a form $B$ if $B(A u, A v)=B(u, v)$ or $B(A u, v)=B(u, A v)$ for all $u$ and $v$.

Each of these problems was considered independently. They were solved in [49, Theorems 3-6] by a unified method over any field $\mathbb{F}$ of characteristic not 2 up to classification of Hermitian forms over finite extensions of $\mathbb{F}$. This gives their full solutions over $\mathbb{R}$ and $\mathbb{C}$ since the classification of Hermitian forms over their finite extensions is known.

The canonical matrices in [49] are given over any field of characteristic not 2, and so they are based on the Frobenius canonical form for similarity. Over $\mathbb{C}$ and $\mathbb{R}$ one can simplify them basing on the Jordan canonical form for similarity. In particular, the canonical matrices of sesquilinear/bilinear forms over $\mathbb{C}$ were simplified in [19] as follows.

Two matrices $M$ and $N$ over $\mathbb{F}$ are called congruent or ${ }^{*}$ congruent if $M=S^{T} N S$ or $M=S^{*} N S\left(S^{*}:=\bar{S}^{T}\right)$ for some nonsingular $S$. Let

$$
\begin{aligned}
& H_{n}(\lambda):=\left[\begin{array}{cc}
0 & I_{n} \\
J_{n}(\lambda) & 0
\end{array}\right] \quad(\lambda \in \mathbb{C}), \\
& \Gamma_{n}:=\left[\begin{array}{ccccc}
0 & & & & \cdot \cdot \\
& & & -1 & . \\
& & 1 & 1 & \\
& -1 & -1 & & \\
1 & 1 & & & 0
\end{array}\right], \quad J_{n}(\lambda):=\left[\begin{array}{cccc}
\lambda & 1 & & 0 \\
& \lambda & \ddots & \\
& & \ddots & 1 \\
0 & & & \lambda
\end{array}\right] \quad \text { (both } n \text {-by- } n \text { ). }
\end{aligned}
$$

For each complex matrix $M$, let us denote by $M^{\mathbb{R}}$ its realification; that is, the real matrix obtained by replacing every entry $a+b i$ of $M$ by the $2 \times 2$ block

$$
\begin{array}{cc}
a & -b \\
b & a
\end{array}
$$


Theorem 3.1. (a) Every square complex matrix is congruent to a direct sum, determined uniquely up to permutation of summands, of matrices of the form

$$
H_{n}(\lambda), \quad \Gamma_{n}, \quad J_{n}(0),
$$

in which $\lambda \neq 0, \lambda \neq(-1)^{n+1}$, and $\lambda$ is determined up to replacement by $\lambda^{-1}$.

(b) Every square complex matrix is *congruent to a direct sum, determined uniquely up to permutation of summands, of matrices of the form

$$
H_{n}(\lambda), \quad \mu \Gamma_{n}, \quad J_{n}(0),
$$

in which $|\lambda|>1$ and $|\mu|=1$.

(c) Every square real matrix is congruent to a direct sum, determined uniquely up to permutation of summands, of matrices of the form:

- $H_{n}(a)$, where $a \in \mathbb{R}, a \neq(-1)^{n+1}$, and $|a| \geqslant 1$;

- $H_{n}(a+b i)^{\mathbb{R}}$, where $a, b \in \mathbb{R}, b>0$, and $a^{2}+b^{2}>1$;

- $\pm \Gamma_{n},\left((a+i) \Gamma_{n}\right)^{\mathbb{R}}$, and $J_{n}(0)$, where $0 \neq a \in \mathbb{R}$.

A direct proof of Theorem 3.1(a,b) is given in [20].

\subsection{Classification problems that can be solved by the linearization method}

The arrows $\gamma$ in (11) and (12) are appended with the only purpose: they ensure the nonsingularity of the forms assigned to $\beta$. In similar cases we will omit $\gamma$ and replace the relations $\gamma \beta=1$ and $\beta \gamma=1$ by $|\beta| \neq 0$. The edges of dographs represent linear mappings and forms. In order to make relations clearer, we will write them as if the edges are mappings and forms; they could be easily rewritten in the form (6).

Theorems 2.1 and 2.2 enable to classify representations of the following dographs $D$ since the classification of representations of the corresponding quivers $\underline{D}$ is known:

(i)

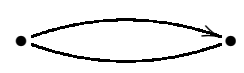

which gives the problem of classifying pairs consisting of a linear mapping from one space to another and a sesquilinear form on these spaces. This problem was solved in [47] (see also Theorem 3.2).

São Paulo J.Math.Sci. 1, 2 (2007), 219-240 
(ii)

$$
\begin{gathered}
\lambda(x, y)= \pm \lambda^{*}(y, x),|\lambda| \neq 0, \\
\mu(x, y)= \pm \mu^{*}(y, x),|\mu| \neq 0, \\
\mu(\alpha x, \alpha y)=\lambda(x, y),
\end{gathered}
$$

which gives the problem of classifying metric mappings from one space with indefinite scalar product to another.

(iii)

$$
\alpha \bigcap_{\mu \quad \lambda(\alpha x, y)= \pm \lambda(x, \alpha y), \mu(\alpha x, y)= \pm \mu(x, \alpha y),}^{\lambda(x, y)= \pm \lambda^{*}(y, x),|\lambda| \neq 0,} \begin{aligned}
\mu(x, y) & = \pm \mu^{*}(y, x),|\mu| \neq 0,
\end{aligned}
$$

which gives the problem of classifying linear operators that are selfadjoint or skew-adjoint with respect to two nonsingular Hermitian/skew-Hermitian forms. This problem was studied in [33].

(iv)

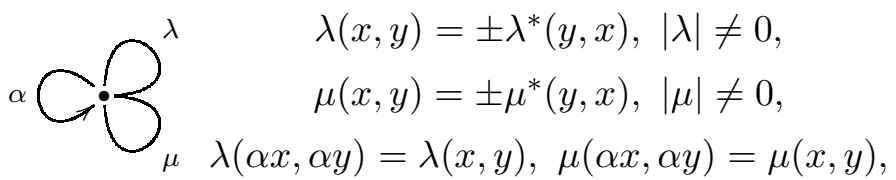

which gives the problem of classifying linear operators that are isometric with respect to two nonsingular Hermitian/skew-Hermitian forms.

(

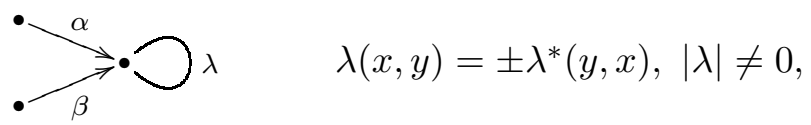

which gives the problem of classifying pairs of subspaces in a space with indefinite scalar product (if we restrict ourselves to those representations $\mathcal{A}$ in which the mappings $\mathcal{A}_{\alpha}$ and $\mathcal{A}_{\beta}$ are injective). This problem was studied in [50]. 
(vi)

$$
\alpha\left(\begin{array}{c}
\lambda(x, y)= \pm \lambda^{*}(y, x),|\lambda| \neq 0, \\
\lambda(\alpha x, \beta y)= \pm \lambda(\beta x, \alpha y),
\end{array}\right.
$$

which gives the problem of classifying selfadjoint/skew-adjoint matrix pencils studied in [26, 29].

(vii)

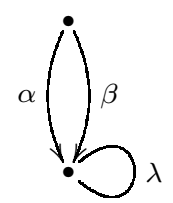

$$
\begin{gathered}
\lambda(x, y)= \pm \lambda^{*}(y, x),|\lambda| \neq 0, \\
\lambda(\alpha x, \alpha y)=\lambda(\beta x, \beta y),
\end{gathered}
$$

which gives the problem of classifying isometric matrix pencils studied in $[10,26,29]$.

\subsection{Tame and wild classification problems}

A quiver is called wild if the problem of classifying its representations does not contain the problem of classifying pairs of matrices up to simultaneous similarity, the other quivers are tame (Drozd's Tame-Wild Theorem $[9])$. The problem of classifying representations of any wild quiver is considered as hopeless since it contains the problems of classifying representations of every quiver, every poset, and every finite dimensional algebra, see, for example, [5].

Theorems 2.1 and 2.2 reduce the problem of classifying representations of a dograph $D$ to the problem of classifying representations of its quiver $\underline{D}$. So we will say that a dograph $D$ is tame or wild if the quiver $\underline{D}$ is tame or, respectively, wild.

The dographs considered in Sections 3.1 and 3.2 are tame.

Examples of wild dographs:

- The dograph

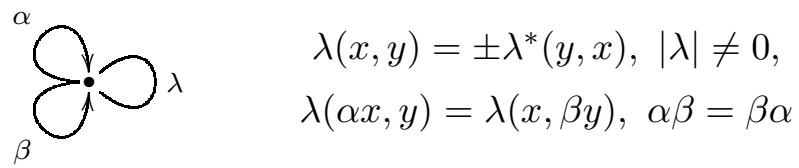

gives the problem (posed in [14, p. 84]) of classifying normal operators on a space with indefinite scalar product. Its wildness was 
proved in [47, Theorem 5.5] and in [15]. Of course, special classes of normal matrices can be classified, see [16, 17, 31, 32].

- The dograph $\alpha C_{1} \supset \lambda$ with relations $\lambda(x, y)= \pm \lambda^{*}(y, x)$ and

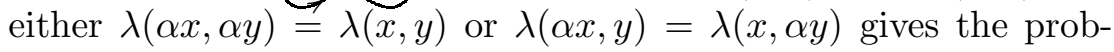
lem of classifying metric or, respectively, selfadjoint linear operators with respect to scalar product that may be singular. Its wildness was proved in [47, Theorem 5.4]. Therefore, the condition of nonsingularity in (11) and (12) is essential.

Each problem of classifying systems of linear mappings and forms some of which are claimed to be Hermitian or skew-Hermitian (symmetric or skew-symmetric if the involution on $\mathbb{F}$ is the identity) can be given by a dograph $D$ in which every nonoriented or doubly oriented loop $\lambda$ is either

- unequipped, or

- equipped with ' + ', which means that there is the relation $\lambda^{*}=\lambda$ on $D$, or

- equipped with ' - ', then there is the relation $\lambda^{*}=-\lambda$,

and there are no other relations on $D$. Such a dograph will be called equipped. For example, the problem of classifying pairs of forms, in which the first is symmetric and the second is skew-symmetric, is given by the equipped dograph $(+-7$.

Theorem 3.2 ([47, Theorem 4.1]). An equipped connected dograph $D$ is tame if and only if it is obtained by orientation of edges

- in one of the Dynkin diagrams

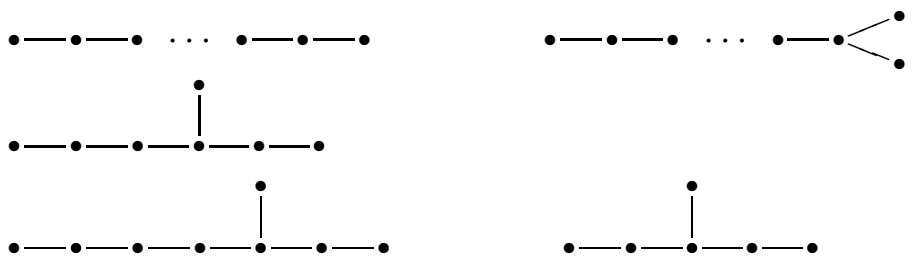

- or in one of the extended Dynkin diagrams
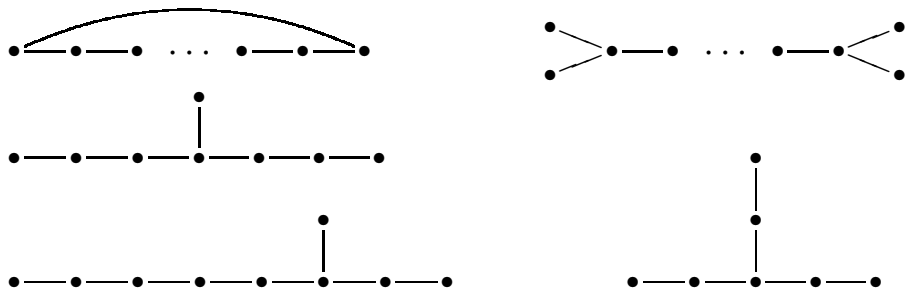
- or in one of the equipped graphs

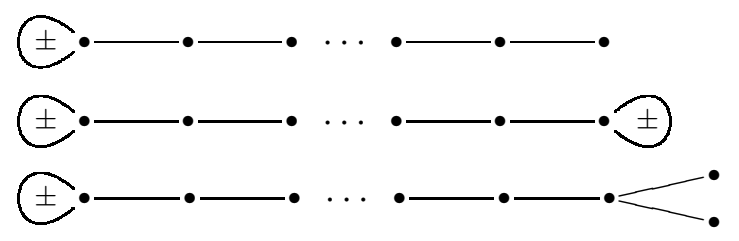

Canonical forms of representations of all dographs obtained by orientations of the edges in (16)-(18) were given in [47] over any field of characteristic not 2 up to classification of Hermitian forms. The proof was based on an analogous result for representations of quivers by Donovan and Freislich [8] and Nazarova [37].

Corollary 3.3. Let $\mathbb{F}$ be an algebraically closed field of characteristic different from 2. The problems of classifying

(i) for each triple $\left(\varepsilon_{1}, \varepsilon_{2}, \varepsilon_{3}\right) \in\{1,-1\}^{3}$, all triples $\left(A_{1}, A_{2}, A_{3}\right)$, up to simultaneous congruence, of $n \times n$ matrices over $\mathbb{F}$ satisfying $A_{i}^{T}=\varepsilon_{i} A_{i}$,

(ii) local algebras over $\mathbb{F}$ with zero cube radical and square radical of dimension 2 ,

(iii) local commutative associative algebras over $\mathbb{F}$ with zero cube radical and square radical of dimension 3 , and

(iv) Lie algebras over $\mathbb{F}$ with central commutator subalgebra of dimension 3

are wild.

The wildness of (i) follows from Theorem 3.2. It implies the wildness of (iii) (resp., of (iv)) since each associative commutative algebra with zero cube radical (resp., Lie algebra with central commutator subalgebra of dimension 3 ) is given by a triple $\left(A_{1}, A_{2}, A_{3}\right)$ of symmetric (resp., skewsymmetric) matrices determined up to simultaneous congruence and up to transformations

$$
\left(A_{1}, A_{2}, A_{3}\right) \mapsto\left(\sum_{i=1}^{3} c_{1 i} A_{i}, \sum_{i=1}^{3} c_{2 i} A_{i}, \sum_{i=1}^{3} c_{3 i} A_{i}\right), \quad \operatorname{det}\left[c_{i j}\right] \neq 0 .
$$

The wildness of (ii)-(iv) was proved in $[3,4]$. Note that the Lie algebras (iv) are also Jordan algebras. 


\subsection{Generalization of Kac's Theorem}

The following generalization of V. Kac's Theorem [23, 24] about the sets of dimensions (see (3)) of indecomposable representations of quivers was proved in [46]. (The positive root system for dographs is defined as for quivers, independently of the orientation of the edges.)

Theorem 3.4. Let us consider representations of a dograph $D$ without relations over an algebraically closed field of characteristic not 2 .

(i) There exists an indecomposable representation of a dograph $D$ of dimension $d$ if and only if $d$ is a positive root of $D$.

(ii) For each positive real root d, there exists a unique up to isomorphism indecomposable representation of dimension $d$.

(iii) For each positive imaginary root d, there exist at least two nonisomorphic indecomposable representations of dimension d. Moreover, for each natural number $n \geqslant 2$, there exist $D$ and $d$ such that there are exactly $n$ nonisomorphic indecomposable representations of $D$ of dimension $d$.

An analogous description of the set of dimensions of indecomposable Euclidean or unitary representations of a quiver (this means that each vertex is assigned by a Euclidean or unitary space) is given in [51].

\section{Systems of tensors as representations of bipartite directed graphs}

Each representation of a dograph is a system of covariant, contravariant, and mixed tensors of rank 2. To include into consideration systems of tensors of arbitrary ranks, we may generalize the notion of a dograph and its representations as follows.

Let $G$ be a bipartite directed graph; that is, a directed graph whose set of vertices decomposed into two disjoint sets $\mathcal{V}$ and $\mathcal{T}$ such that no arrow connects two vertices within the same set. Vertices of $\mathcal{V}$ and $\mathcal{T}$ are represented, respectively, by $\bullet$ and $\circ$. A representation of $G$ over a field $\mathbb{F}$ is given by assigning

- to each vertex $v \in \mathcal{V}$, a vector space $V$ over $\mathbb{F}$, and

- to each vertex $t \in \mathcal{T}$ with $p+q$ arrows

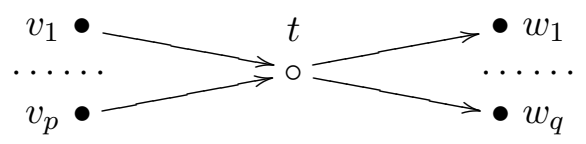


(some of the vertices $v_{1}, \ldots, v_{p}, w_{1}, \ldots, w_{q}$ may coincide), a tensor

$$
T \in V_{1}^{*} \otimes \cdots \otimes V_{p}^{*} \otimes W_{1} \otimes \cdots \otimes W_{q},
$$

which can be identified with the corresponding linear mapping

$$
\begin{gathered}
T: V_{1} \otimes \cdots \otimes V_{p} \longrightarrow W_{1} \otimes \cdots \otimes W_{q} \\
\left(V_{1} \otimes \cdots \otimes V_{p} \text { is } \mathbb{F} \text { if } p=0\right) .
\end{gathered}
$$

For example, each representation of the bipartite directed graph

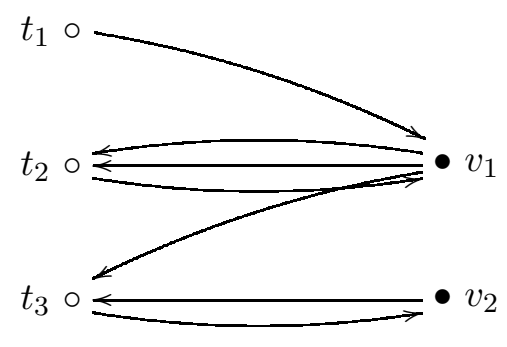

consists of two vector spaces $V_{1}$ and $V_{2}$ and three tensors

$$
T_{1} \in V_{1}, \quad T_{2} \in V_{1}^{*} \otimes V_{1}^{*} \otimes V_{1}, \quad T_{3} \in V_{1}^{*} \otimes V_{2}^{*} \otimes V_{2} .
$$

Each pair $(\Lambda, M)$ consisting of a finite dimensional algebra $\Lambda$ over $\mathbb{F}$ and a module $M$ over $\Lambda$ defines the following representation of the bipartite directed graph (19):

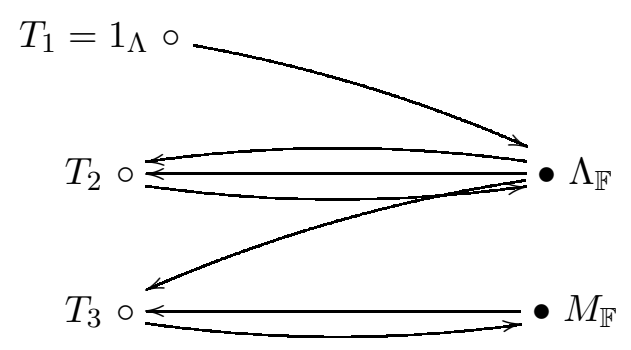

where $\Lambda_{\mathbb{F}}$ and $M_{\mathbb{F}}$ are the underlying vector spaces of $\Lambda$ and $M, T_{1}$ is the identity of $\Lambda$, and the tensors

$$
T_{2}=\sum \lambda_{i 1}^{*} \otimes \lambda_{i 2}^{*} \otimes \lambda_{i 3}, \quad T_{3}=\sum \lambda_{i}^{*} \otimes m_{i 1}^{*} \otimes m_{i 2}
$$

São Paulo J.Math.Sci. 1, 2 (2007), 219-240 
define multiplications in $\Lambda$ and $M$ :

$$
\left(\lambda^{\prime}, \lambda^{\prime \prime}\right) \mapsto \sum\left(\lambda_{i 1}^{*} \lambda^{\prime}\right)\left(\lambda_{i 2}^{*} \lambda^{\prime \prime}\right) \lambda_{i 3}, \quad(\lambda, m) \mapsto \sum\left(\lambda_{i 1}^{*} \lambda\right)\left(m_{i 1}^{*} m\right) m_{i 2}
$$

The identities (additivity, distributivity, ... ) defining the algebra and module structures can be written using tensor contractions. This example leads us to the problem of studying representations of bipartite directed graphs with relations that are linear combinations of tensor products and contractions.

Note that the set of representations of each quiver or dograph can be identified with the set of representations of the corresponding bipartite directed graph $G$, in which $(p, q)=(1,1)$ or, respectively, $p+q=2$ for every vertex $t \in \mathcal{T}$.

The following generalization of Sylvester's Inertia Theorem was proved in [6] for $n$-linear forms with $n \geqslant 2$.

Theorem 4.1. If $G$ is a bipartite directed graph in which every vertex $t \in \mathcal{T}$ has at least two arrows (that is, each representation consists of tensors of rank $\geqslant 2$ ), then every representation of $G$ over $\mathbb{R}$ or $\mathbb{C}$ decomposes into a direct sum of indecomposable representations uniquely up to isomorphism of summands.

Each $n$-linear form $T: V \times \cdots \times V \rightarrow \mathbb{F}$ defines

- the representation $\mathcal{P}=(T, V)$ of the bipartite directed graph

$G:$

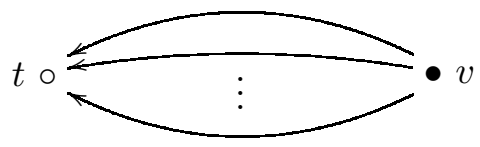

( $n$ arrows $)$

and

- the representation $\overline{\mathcal{P}}=\left(T_{1}, \ldots, T_{n !}, V, \ldots, V\right)$ with

$$
T_{i}\left(v_{1}, \ldots, v_{n}\right):=T\left(v_{\sigma_{i}(1)}, \ldots, v_{\sigma_{i}(n)}\right)
$$


(here $S_{n}=\left\{\sigma_{1}, \ldots, \sigma_{n !}\right\}$ is the set of all substitutions on $1, \ldots, n$ ) of the bipartite directed graph

$\bar{G}$ :

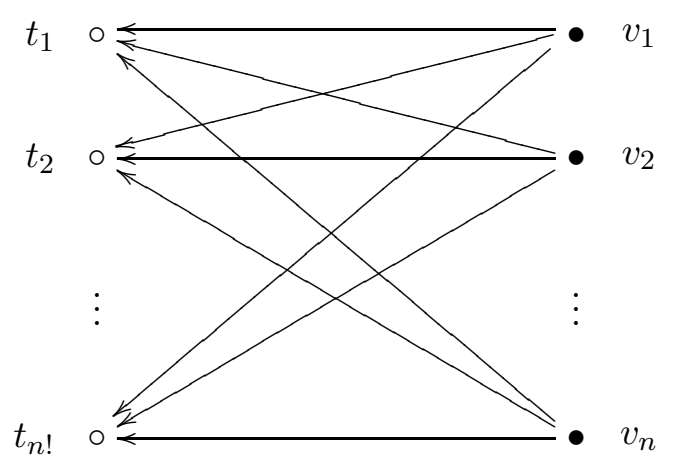

The following statement generalizes (1) and is a reformulation of $[6$, Theorem 2].

Theorem 4.2. Let $G$ and $\bar{G}$ be the bipartite directed graphs (20) and (21).

(a) Two representations $\mathcal{P}$ and $\mathcal{Q}$ of $G$ over $\mathbb{C}$ (i.e., two n-linear forms) are isomorphic if and only if the corresponding representations $\overline{\mathcal{P}}$ and $\overline{\mathcal{Q}}$ of $\bar{G}$ are isomorphic.

(b) Let $\mathcal{P}=\mathcal{P}_{1} \oplus \cdots \oplus \mathcal{P}_{s} \oplus 0$ and $\mathcal{Q}=\mathcal{Q}_{1} \oplus \cdots \oplus \mathcal{Q}_{r} \oplus 0$ be two representations of $G$ over $\mathbb{R}$, in which all summands $\mathcal{P}_{i}$ and $\mathcal{Q}_{j}$ are nonzero and direct-sum-indecomposable. Let the corresponding representations $\overline{\mathcal{P}}$ and $\overline{\mathcal{Q}}$ of $\bar{G}$ be isomorphic. Then $s=r$ and, after a suitable reindexing, $\mathcal{P}_{i}$ is isomorphic to $\pm \mathcal{Q}_{i}$.

\section{Systems of linear and semilinear mappings}

A mapping $A$ from a complex vector space $U$ to a complex vector space $V$ is called semilinear and is denoted by $A: U \rightarrow V$ if

$$
A\left(u_{1}+u_{2}\right)=A u_{1}+A u_{2}, \quad A(\alpha u)=\bar{\alpha} A u
$$

for all $u_{1}, u_{2}, u \in U$ and $\alpha \in \mathbb{C}$. Each semilinear mapping $A: U \rightarrow V$ defines the linear mapping $A: U \rightarrow \bar{V}$, where the conjugate vector space $\bar{V}$ has the same additive group as $V$ and the multiplication by $\alpha \in \mathbb{C}$ in $V$ coincides with the multiplication by $\bar{\alpha}$ in $\bar{V}$. If $A_{f e}$ is the matrix of $A$ in some bases $\left\{e_{i}\right\}$ of $U$ and $\left\{f_{j}\right\}$ of $V$, then the coordinate vector of $A u$ is $A_{f e} \overline{[u]}_{e}$, where $[u]_{e}$ is the coordinate vector of $u \in U$. The matrix of $A$ in 
other bases is $R^{-1} A_{f e} \bar{S}$, where $R$ and $S$ are the transition matrices (see $[7])$.

The following statement by Horn and Merino [18, Theorem 30] resembles (1):

square complex matrices $M$ and $N$ are consimilar if and only if the pairs $(M, \bar{M})$ and $(N, \bar{N})$ are simultaneously equivalent.

(Two complex matrices $M$ and $N$ are called consimilar if $M=\bar{S}^{-1} N S$ for some nonsingular $S$.) Therefore, the canonical form problem for matrices of semilinear operators reduces to the canonical form problem for matrix pencils.

Let us extend (22) to systems of linear and semilinear mappings in the same way as (1) was extended in Section 2 to systems of linear mappings and forms.

A directed graph with usual and dashed arrows will be called a biquiver. Its representation is given by assigning to each vertex a complex vector space, to each usual arrow a linear mapping, and to each dashed arrow a semilinear mapping. For example, a representation

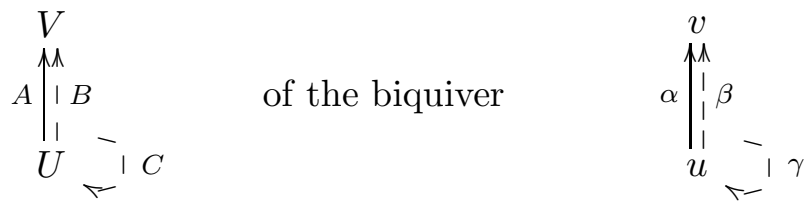

is formed by a linear mapping $A: U \rightarrow V$ and semilinear mappings $B: U \rightarrow V$ and $C: U \rightarrow U$.

For every biquiver $Q$, we construct the quiver $\underline{Q}$ with involution by replacing

- each vertex $u$ by two vertices $u, \bar{u}$,

- each arrow $\alpha: u \rightarrow v$ by two arrows $\alpha: u \rightarrow v, \bar{\alpha}: \bar{u} \rightarrow \bar{v}$,

- each dashed arrow $\beta: u \rightarrow v$ by two arrows $\beta: u \rightarrow \bar{v}, \bar{\beta}: \bar{u} \rightarrow v$.

Every representation $\mathcal{R}$ of $Q$ defines the "self-conjugate" representation $\underline{\underline{\mathcal{R}}}$ of $Q$ since each mapping $A: U \rightarrow V$ defines the linear mapping $\bar{A}: \bar{U} \rightarrow \bar{V}$ and each semilinear mapping $B: U \rightarrow V$ defines the linear mappings $B: U \rightarrow \bar{V}$ and $\bar{B}: \bar{U} \rightarrow V$. For example, the biquiver and its representation (23) define the following quiver $\underline{Q}$ with involution and its representation 
$\underline{\mathcal{R}}:$

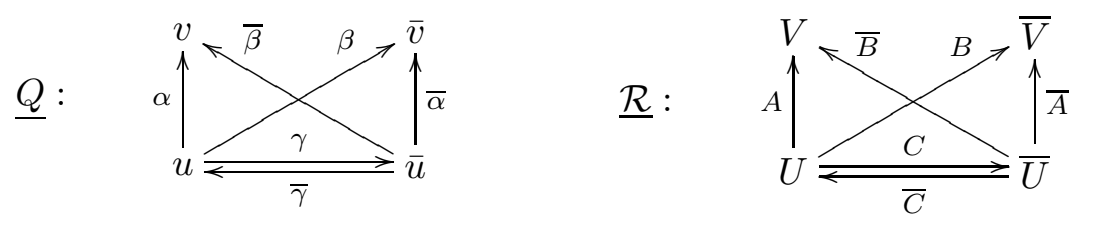

The following theorem generalizes (22) and reduces the theory of systems of linear and semilinear mappings to the theory of "self-conjugate" systems of linear mappings.

Theorem 5.1. Two representations $\mathcal{R}$ and $\mathcal{R}^{\prime}$ of a biquiver $Q$ are isomorphic if and only if the representations $\underline{\mathcal{R}}$ and $\underline{\mathcal{R}}^{\prime}$ of the quiver $\underline{Q}$ are isomorphic.

\section{References}

[1] D. Alpay, A. C. M. Ran, L. Rodman, Basic classes of matrices with respect to quaternionic indefinite inner product spaces, Linear Algebra Appl. 416 (2006) 242-269.

[2] Y.-H. Au-Yeung, C.-K. Li, L. Rodman, H-unitary and Lorentz matrices: A review, SIAM J. of Matrix Analysis, 25 (2004) 1140-1162.

[3] G. Belitskii, V. M. Bondarenko, R. Lipyanski, V. V. Plachotnik, V. V. Sergeichuk, The problems of classifying pairs of forms and local algebras with zero cube radical are wild, Linear Algebra Appl. 402 (2005) 135-142.

[4] G. Belitskii, R. Lipyanski, V. V. Sergeichuk, Problems of classifying associative or Lie algebras and triples of symmetric or skew-symmetric matrices are wild, Linear Algebra Appl. 407 (2005) 249-262.

[5] G. R. Belitskii, V. V. Sergeichuk, Complexity of matrix problems, Linear Algebra Appl. 361 (2003) 203-222.

[6] G. R. Belitskii, V. V. Sergeichuk, Congruence of multilinear forms, Linear Algebra Appl. 418 (2006) 751-762.

[7] N. Bourbaki, Elements of Mathematics, Algebra I, Springer, 1989.

[8] P. Donovan, M. R. Freislich, The representation theory of finite graphs and associated algebras, Carleton Math. Lecture Notes 5, Ottawa, 1973.

[9] Yu. A. Drozd, Tame and wild matrix problems, Lect. Notes Math. 832 (1980) 242-258.

[10] A. Ferrante, B. C. Levy, Canonical form of symplectic matrix pencils, Linear Algebra Appl. 274 (1998) 259-300.

[11] P. Gabriel, Unzerlegbare Darstellungen I, Manuscripta Math. 6 (1972) 71-103.

[12] P. Gabriel, Appendix: degenerate bilinear forms, J. Algebra 31 (1974) 67-72.

[13] P. Gabriel, A. V. Roiter, Representations of Finite-Dimensional Algebras, Springer-Verlag, 1997. 
[14] I. Gohberg, P. Lancaster, L. Rodman, Matrices and Indefinite Scalar Products, Birkhäuser-Verlag, Basel, Switzerland, 1983.

[15] I. Gohberg, B. Reichstein, On classification of normal matrices in an indefinite scalar product, Integral Equations and Operator Theory 13 (1990) 364-394.

[16] O. Holtz, V. Strauss, Classification of normal operators in spaces with indefinite scalar product of rank 2, Linear Algebra Appl. 241-243 (1996) 455-517.

[17] O. Holtz, V. Strauss, On classification of normal operators in real spaces with indefinite scalar product, Linear Algebra Appl. 255 (1997) 113-155.

[18] R. A. Horn, D. I. Merino, Contragredient equivalence: a canonical form and some applications, Linear Algebra Appl. 214 (1995) 43-92.

[19] R. A. Horn, V. V. Sergeichuk, Congruence of a square matrix and its transpose, Linear Algebra Appl. 389 (2004) 347-353.

[20] R. A. Horn, V. V. Sergeichuk, Canonical forms for complex matrix congruence and *congruence, Linear Algebra Appl. 416 (2006) 1010-1032.

[21] B. Huppert, Isometrien von Vektorräumen. I, Arch. Math. (Basel) 35 (1980) 164176.

[22] B. Huppert, Isometrien von Vektorräumen. II, Math. Z. 175 (1980) 5-20.

[23] V. G. Kac, Infinite root systems, representation of graphs and invariant theory, Invent. Math. 56 (1980) 57-92.

[24] V. G. Kac, Infinite root systems, representation of graphs and invariant theory, II, J. Algebra 78 (1982) 141-162.

[25] M. Karow, Self-adjoint operators and pairs of Hermitian forms over the quaternions, Linear Algebra Appl. 299 (1999) 101-117.

[26] I. Krupnik, P. Lancaster, $H$-selfadjoint and $H$-unitary matrix pencils, SIAM J. Matrix Anal. Appl. 19 (no. 2) (1998) 307-324.

[27] P. Lancaster, L. Rodman, Canonical forms for symmetric/skew-symmetric real matrix pairs under strict equivalence and congruence, Linear Algebra Appl. 406 (2005) 1-76.

[28] P. Lancaster, L. Rodman, Canonical forms for Hermitian matrix pairs under strict equivalence and congruence, SIAM Rev. 47 (no. 3) (2005) 407-443.

[29] W.-W. Lin, V. Mehrmann, H. Xu, Canonical forms for Hamiltonian and symplectic matrices and pencils, Linear Algebra Appl. 301-303 (1999) 469-533.

[30] A. I. Mal'cev, Foundations of Linear Algebra, W. H. Freeman \& Co., San Francisco, 1963.

[31] C. Mehl, On classification of normal matrices in indefinite inner product spaces, Electron. J. Linear Algebra 15 (2006) 50-83.

[32] C. Mehl, Essential decomposition of polynomially normal matrices in real indefinite inner product spaces, Electron. J. Linear Algebra 15 (2006) 84-106.

[33] C. Mehl, V. Mehrmann, H. Xu, Canonical forms for doubly structured matrices and pencils, Electron. J. Linear Algebra 7 (2000) 112-151.

[34] C. Mehl, L. Rodman, Symmetric matrices with respect to sesquilinear forms, Linear Algebra Appl. 349 (2002) 55-75. 
[35] V. Mehrmann, H. Xu, Structured Jordan canonical forms for structured matrices that are Hermitian, skew Hermitian or unitary with respect to indefinite inner products, Electron. J. Linear Algebra 5 (1999) 67-103.

[36] J. Milnor, On isometries of inner product spaces, Invent. Math. 8 (1969) 83-97.

[37] L. A. Nazarova, Representations of quivers of infinite type, Math. USSR-Izv. 7 (1973) 749-792.

[38] C. Riehm, The equivalence of bilinear forms, J. Algebra 31 (1974) 45-66.

[39] C. Riehm, M. Shrader-Frechette, The equivalence of sesquilinear forms, J. Algebra 42 (1976) 495-530.

[40] L. Rodman, Similarity vs unitary similarity and perturbation analysis of sign characteristic: Complex and real indefinite inner products, Linear Algebra Appl. 416 (2006) 945-1009.

[41] A. V. Roiter, Bocses with involution, in: Representations and Quadratic Forms, Akad. Nauk Ukrain. SSR, Inst. Mat., Kiev, 1979, 124-128 (in Russian).

[42] R. Scharlau, Paare alternierder Formen, Math. Z. 147 (1976) 13-19.

[43] R. Scharlau, Zur Klassification von Bilinearformen und von Izometrien über Körpern, Math. Z. 178 (1981) 359-373.

[44] W. Scharlau, Quadratic and Hermitian Forms, Springer-Verlag, 1985.

[45] V. V. Sergeichuk, Representations of simple involutive quivers, in: Representations and quadratic forms, Akad. Nauk Ukrain. SSR, Inst. Mat., Kiev, 1979, pp. 127-148 (in Russian).

[46] V. V. Sergeichuk, Representations of directed schemes, in: Linear Algebra and Theory of Representations, Akad. Nauk Ukrain. SSR, Inst. Mat., Kiev, 1983, pp. 110-134 (in Russian).

[47] V. V. Sergeichuk, Classification problems for systems of linear mappings and sesquilinear forms (Russian) Preprint, Kiev University, 1983, 60 p. = Manuscript No. 196 Uk-D84, deposited at the Ukrainian NIINTI, 1984; R. Zh. Mat. 1984, 7A331.

[48] V. V. Sergeichuk, The canonical form of the matrix of a bilinear form over an algebraically closed field of characteristic 2, Math. Notes 41 (no. 5-6) (1987) 441-445.

[49] V. V. Sergeichuk, Classification problems for systems of forms and linear mappings, Math. USSR-Izv. 31 (no. 3) (1988) 481-501.

[50] V. V. Sergeichuk, Classification of pairs of subspaces in scalar product spaces, Ukrainian Math. J. 42 (no. 4) (1990) 487-491.

[51] V. V. Sergeichuk, Unitary and Euclidean representations of a quiver, Linear Algebra Appl. 278 (1998) 37-62.

[52] R. C. Thompson, Pencils of complex and real symmetric and skew matrices, Linear Algebra Appl. 147 (1991) 323-371.

[53] F. Uhlig, A recurring theorem about pairs of quadratic forms and extensions: a survey, Linear Algebra Appl. 25 (1979) 219-237.

[54] F. Uhlig, A rational canonical pair form for a pair of symmetric matrices over an arbitrary field $F$ with char $F \neq 2$ and applications to finest simultaneous block diagonalizations, Linear Multilinear Algebra 8 (1979/80) 41-67.

[55] W. C. Waterhouse, Pairs of quadratic forms, Invent. Math. 37 (no. 2) (1976) $157-164$. 